\title{
Personalized Forecasting System for Complex Product Quality Based on Predictive Control Model of Multiple Linear Regression Analysis
}

\author{
Jihong Pang \\ College of Mechanical and Electronic Engineering, Wenzhou University, \\ Wenzhou 325035, Zhejiang, China \\ pangjihong@163.com
}

\begin{abstract}
Forecasting quality of complex products is a major concern for quality engineers and enterprises' decision-makers. But only a few researchers have investigated how multiple linear regression analysis can improve forecasts. This article presents a predictive control model for personalized forecasting system of complex product quality using multiple linear regression analysis. First, this paper compares the performance of personalized forecasting system for product quality which leads to a better understanding of the applicability of the forecasting model. Second, this paper identifies the effect of predictive control model of multiple linear regression analysis on forecasting accuracy. Then, an example shows the feasibility of using predictive control model in complex product quality personalized forecasting. Finally, it makes a conclusion that the approved prediction system embraces superiority over the prediction of the quality factors and manufacturing resources.
\end{abstract}

Keywords: Product Quality; Forecasting System; Complex Product; Predictive Control Model; Multiple Linear Regression Analysis

\section{Introduction}

Complex product quality forecasting is an arduous task for researchers, academics and practitioners. So, the ability to predict product quality has drawn considerable attention from academic researchers [1]. In addition, the scientific forecasting system of complex product quality is an important basis in the design and development process and in manufacturing process activities. In the meantime, complex product quality forecast is very important to the rational utilization and distribution of enterprise resources. Product quality forecasting is an effective means for the planning and management of quality managers [2]. It is also the most important foundation work of optimizing the allocation of enterprise resources. In order to assess the risks that they face, many enterprises require an estimation of the likelihood, timing, and extent of possible economically related loss for each of their cooperative businesses. Moreover, veracious forecast of the actual product quality level is very significant for the programming of enterprise resource [3]. An unpredicted change that occurs in one part of the manufacturing enterprises will rapidly spread to other parts of the enterprises and bring risks to business operations. Due to increasing awareness of the adverse consequences of product failures, numerous enterprises have promulgated product quality standards [4]. Thus, it should be remembered that the fundamental purpose of the quality policy is to allow industrial growth despite stringent product quality standards. Adherence to the sustainable development strategies, manufacturing enterprises should increase forecasting accuracy and reduce risks with the central objectives of improving the product quality to meet the customer requirements [5]. In a word, the local manufacturing enterprises at various 
levels shall be responsible for product quality under their jurisdiction and take measures to improve the product quality [6].

Meanwhile, the lots of enterprises have intensified product quality protection. As stipulated in laws of most countries and areas, the product quality standards are compulsory standards, and those who violate these compulsory quality standards must bear the corresponding legal responsibility [7]. With the fast economic development in world, breakthroughs were also made in the prevention and control of product quality, the improvement of product quality and the strengthening of quality guarantee system. Above all, the personalized forecasting system has been introduced, which helped improve the product quality and decline product cost. In accordance with the strategy of development of different branches of the products, the personalized forecasting system for complex product quality based on predictive control model of multiple linear regression analysis was presented in this article. By use of this personalized forecasting system, the new information can be inputted continually to predict the quality of complex product in the mechanical and intellectual making [8]. Besides that, a reliable failure prediction model normally gives a longer period of advance warning signals prior to the actual event. The advance warning signals enable both managers and technical personnel to take appropriate preventive actions including operating policy changes. And for this reason, we aimed to determine key characteristics of signals that would allow prediction of those enterprises that are at highest risk for product quality.

More important, some studies have shown that a number of quantitative and qualitative models for analyzing and forecasting the product quality have been developed. There are, however, several methods for improving the predictive ability by relying upon realistic data. In order to improve the ability to predict early failure for product quality, multiple linear regression analysis could be an alternative solution [9]. Increased investment in forecasting system has played a positive role in controlling product failures and improving product quality. So product quality forecasting is the basis of the rational exploitation, management, quality control and programming in the whole process of manufacturing and service providing. The regressive analysis is that the data of engineering and scientific experiment are fitted into linear function to correspond to the mutual relationship among the variables of personalized forecasting system [10]. Thus, we aimed to describe the personalized forecasting system and develop a predictive model of complex product quality serious using multiple linear regression analysis.

On the basis of this rationale, the purpose of this article is to present a framework for a predictive control model based on early warning information by using multiple linear regression analysis. Although we must continue to work on predictive model, substantial results have been achieved, including remarkable improvement in product quality through manufacturing process controls and greater use of the advanced prediction system. Thus, this paper is structured as follows: In Chapter 2, the application and recent research progress of personalized forecasting system for product quality are introduced. Chapter 3 explains the predictive control model of multiple linear regression analysis. A numerical example is given to illustrate the applicability of this condition in Chapter 4. Finally, we get some useful conclusions and give some suggestion.

\section{Personalized Forecasting System for Product Quality}

Prediction of product quality is the major part of the technology. This is a new attempt on the forecast for the change tendency of product quality. As the important part of manufacturing resource planning and management, product quality evaluation and forecast are of vital realistic significance to promote sustainable utilization [11]. Basing on present product quality and future developmental tendency, supporting capacity of enterprise resources will be a key factor on sustainable development of economy and industrial enterprises. Meanwhile, resource shortages and product quality problems are 
also the legacy during the technology progress of manufacturing industry[12]. If the prediction can be done reasonably and precisely, it can avoid waste and occurrence product supply crisis. It is planned that personalized forecasting system will be devoted to improve the product quality significantly and improve the quality control preliminarily. And these variables of personalized forecasting system dealt with defects, mistakes and symptoms of failure. The personalized forecasting system for complex product quality is presented in Figure 1.

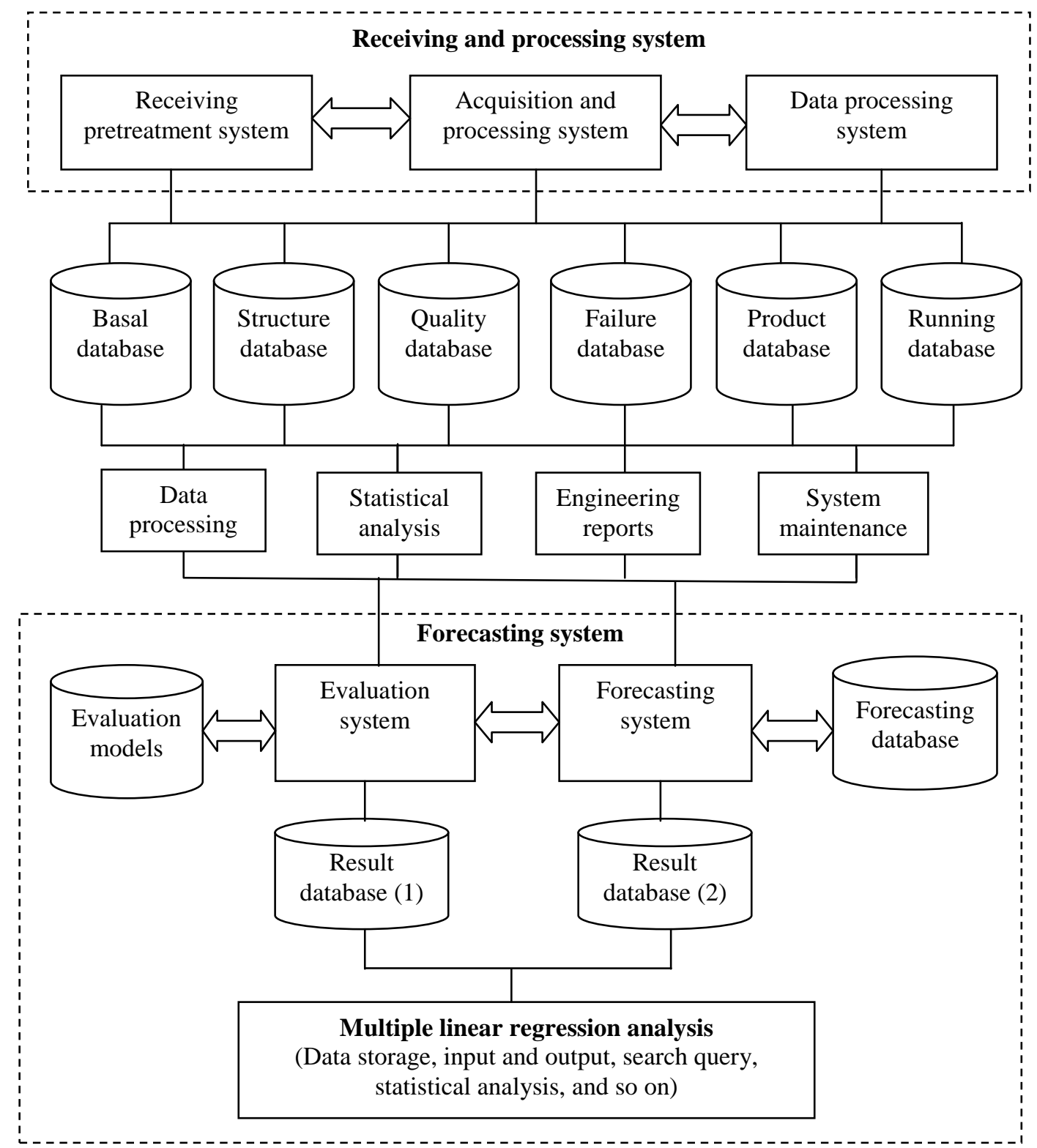

Figure 1. Personalized Forecasting System for Product Quality

The difficulties in the implement of personalized forecasting system and the burning desire to improve the quality among common people are the main causes. Likewise, we can use personalized forecasting system in quality management work to improve the product quality. Many fields of manufacturers' application in assessment and forecasting of product quality system are studied in this paper. In some areas, local enterprises are encouraging information technology talents to start telecommuting programs in order to reduce quality costs and improve product quality. The results of studies indicate that 
personalized forecasting system not only improve product quality but can boost productivity and reduce the amount of defective products. The total discharge of major product quality problems have decreased steadily and the product quality in key areas has been improving. Through leadership and actions, top management can create a personalized forecasting system which workers are fully involved and in which a quality management system can operate effectively.

\section{Predictive Control Model of Multiple Linear Regression Analysis}

Now normally, multiple linear regression analysis is always used to measure the relevance of the data information. Analyses of personalized forecasting system were achieved by multiple linear regression analysis and logistic regression analysis [13]. Product quality can be evaluated and forecasted by using multiple linear regression analysis method [14]. Based on the summarization of present research situation of multiple linear regression analysis theory for product quality, a new predictive control model was established in this paper.

Next, a predictive control model of product quality is built, and the results show that partial least-square regression is more capable of building control models reflecting correct relations among several variables compared with normal multi-variable regression method. On the basis of a lot of experimental data, experimental mathematical models of predictive control model of several sorts of fault characteristics are built up with multiple linear regression analysis [15]. Using predictive control model of multiple linear regression analysis, product quality has been forecasted synthetically [16]. More important, regression process contains the likelihood of future values of the dependent variable being in error [17]. When applying regression analysis, people sometimes want to find a relationship between two variables [18]. Then, the sensitivity and specificity of the fault factors diagnostic test were analyzed [19]. Multiple linear regression analysis was used to measure the relevance of information [20].

Given the above, linear equations for product quality and related parameters were established upon multiple linear regression analysis, providing a math model for optimum design of personalized forecasting system. Our goal is to take predictive problems and break them down into these computational steps, these sequence of instructions that'll allow us to capture that process. The method of multiple linear regression analysis is given in subsequent sections.

Firstly, many Yuan linear return forecast model is given by:

$y=a_{0}+a_{1} x_{1}+a_{2} x_{2}$

Where, $\mathrm{x} 1$ and $\mathrm{x} 2$ represent the influence factor of independent variables; $\mathrm{y}$ represents the predicted values of dependent variables; $a 0$, a1 and a2 represent initial values.

In the ordinary circumstances, it turns the center value optimization question into the boundary value question of the least square problem to process. Then it calls for constructing the discriminant function of the form:

$$
\begin{aligned}
& y^{\prime}=y-\bar{y} \\
& x_{1}^{\prime}=x_{1}-\bar{x}_{1} \\
& x_{2}^{\prime}=x_{2}-\bar{x}_{2}
\end{aligned}
$$

So, the equation for calculating the center value is presented, which can be used for calculating the change value. 
$y^{\prime}=a_{1} x_{1}^{\prime}+a_{2} x_{2}^{\prime}$

Then, the object data can be analyzed and processed by using least-square theory. By using the linear least-square fitting method for evaluation of system parameters and inspected through square error analysis and $\mathrm{F}$ test, a rate equation is proposed in this paper. The formula is shown as the following:

$a_{1}=\frac{\sum x_{1}^{\prime} y^{\prime} \cdot \sum\left(x_{2}^{\prime}\right)^{2}-\sum x_{1}^{\prime} x_{2}^{\prime} \cdot \sum x_{2}^{\prime} y^{\prime}}{\sum\left(x_{1}^{\prime}\right)^{2} \cdot \sum\left(x_{2}^{\prime}\right)^{2}-\sum x_{1}^{\prime} x_{2}^{\prime} \cdot \sum x_{1}^{\prime} x_{2}^{\prime}}$

Similarly, the other general coefficient of forecasting equation by can be computed by the following equation:

$a_{2}=\frac{\sum x_{2}^{\prime} y^{\prime} \cdot \sum\left(x_{1}^{\prime}\right)^{2}-\sum x_{1}^{\prime} x_{2}^{\prime} \cdot \sum x_{1}^{\prime} y^{\prime}}{\sum\left(x_{1}^{\prime}\right)^{2} \cdot \sum\left(x_{2}^{\prime}\right)^{2}-\sum x_{1}^{\prime} x_{2}^{\prime} \cdot \sum x_{1}^{\prime} x_{2}^{\prime}}$

Next, the predicted data are then normalized as:

$a_{0}=\bar{y}-a_{1} \bar{x}_{1}+a_{2} \bar{x}_{2}$

The model is tested by taking the predicted values, and the relatively errors of the theoretical values and the real record are presented. Model checking is an important way in developing high confidence forecasting system. The miscellaneous model inspection in the general regressive modeling can be avoided and the model parameters can be decided simultaneously and effectively. Finally, we find support for a forecast model examining mechanisms driving the effect of standard precision on aggressive reporting decisions. Thus, the multiple correlation coefficient of the model is obtained by the following equation:

$R=\sqrt{1-\frac{\sum(y-\hat{y})^{2}}{\sum(y-\bar{y})^{2}}}$

Where $\mathrm{R}$ is the measured data, $0 \leq R \leq 1 . \hat{y}$ represents the predicting result of forecasting model.

To reduce computational complexity effectively, this paper transforms the consistency checking problem into a general statistical hypothesis testing problem. Statistical analyses for hypothesis testing were carried on. Then, we introduce the method for testing statistical hypothesis. The measured data of F testing are then normalized as:

$$
F=\frac{R^{2} / k}{\left(1-R^{2}\right) /(n-k-1)}
$$

Where $\mathrm{k}$ represents the exact quantity of independent variables, $\mathrm{n}$ represents the exact quantity of measured values. Under a certain significant level of $\alpha$, F $\alpha$ can be computed by using database table look-up techniques. So we have a hypothesis. If $F \leq F_{\alpha}$, the correlative test is no a close relationship possibility, otherwise the parameters of the model should be recalculated.

\section{Numerical Example}

According to the actual situation of complex product quality, this paper predicts the development of status of enterprises competitiveness, and forecasts the products 
performance on this base. In order to apply the method for the product quality and enterprise resources field, this paper sets the computer numerical control (CNC) machining center as an example by using multiple linear regression analysis. More significantly, the numerical example of CNC machining center includes some of the major multi-variable tests, a single variable $\mathrm{F}$ tests, trend analysis and multiple linear regression analysis. Started from the current situation of quality of CNC machining center, the developmental tendency of enterprise resources was forecasted by dynamic simulation.

In this article we verify the hypotheses by multiple linear regression, analysis of variance, factor analysis and correlation analysis based on personalized forecasting system. After stepwise multiple regression analysis, predicted variables contributing to reliability of system were found to be associated with mean time between failures (MTBF) and product development lifecycle. Multiple linear regression and correlative analysis showed that the increase in reliability of system of CNC machining center was main factor for the formation of quality for $\mathrm{CNC}$ machining center. Through multiple linear regression and correlative analysis, we obtain the relevant equation of the quality characters and the sensory index of MTBF and product development lifecycle. The values of statistics database of CNC machining center is shown in Table 1.

Table 1. The Values of Statistics Database of CNC Machining Center

\begin{tabular}{c|c|c|c}
\hline \multirow{2}{*}{ No. } & Reliability of system & MTBF & Product development lifecycle \\
\cline { 2 - 4 } & Percentage $(y)$ & Hour $\left(x_{1}\right)$ & Month $\left(x_{2}\right)$ \\
\hline 1 & 97.5 & 1521 & 20 \\
\hline 2 & 96.4 & 1432 & 16 \\
\hline 3 & 93.5 & 1320 & 17 \\
\hline 4 & 97.6 & 1550 & 28 \\
\hline 5 & 97.8 & 1644 & 18 \\
\hline 6 & 91.2 & 1358 & 23 \\
\hline 7 & 93.1 & 1348 & 15 \\
\hline 8 & 91.5 & 1265 & 19 \\
\hline 9 & 94.6 & 1350 & 18 \\
\hline 10 & 95.4 & 1400 & 15 \\
\hline 11 & 93.2 & 1385 & 16 \\
\hline 12 & 91.5 & 1315 & 18 \\
\hline 13 & 93.4 & 1768 & 26 \\
\hline 14 & 94.5 & 1342 & \\
\hline 15 & 94.5 & 1623 & \\
\hline
\end{tabular}

In this paper, the panel data of $\mathrm{CNC}$ machining center was cited to make a multiple linear regression analysis. According to the features of the CNC machining center, there were two different types of predictive control models for the product quality set up. The product quality can be quantitatively predicted with multiple linear regression analysis. Based on the daily demand level and precipitation data during in producing and manufacturing process and the variation features of enterprise resources have been analyzed. These models have been successful in providing a systematic procedure for the analysis of product quality, but their success in forecasting unexpected quality factor changes has been somewhat limited. By multiple linear regression analysis, the quantitative relationship between reliability of system, MTBF and product development lifecycle were determined. 
In this case, each member of decision makers has full knowledge of the company which he or she is responsible for and could narrate events leading to the reliability of system. By comparing different methods, factor analysis and multiple linear regression analysis were used to calculate the reliability of system of CNC machining center. Based on facts of CNC machining center, this paper analyses enterprise resource quantity and supplying quantity, forecasts product demand in different years and analyses product quantity balance.

By using Equation (2), we can get the discriminant function in the following:

$$
\bar{y}=\sum_{i=1}^{15} y_{i}=93.48
$$

And the same method can be applied to the other discriminant functions. The way these methods work under the covers is also important and should be taken into account when developing our application. The statistical data set of multiple linear regression analysis is given in Table 2.

Table 2. The Statistical Data Set of Multiple Linear Regression Analysis

\begin{tabular}{c|c|c|c|c|c|c|c|c}
\hline No. & $y^{\prime}$ & $x_{1}^{\prime}$ & $x_{2}^{\prime}$ & $x_{1}{ }^{\prime} x_{1}^{\prime}$ & $x_{2}{ }^{\prime} x_{2}{ }^{\prime}$ & $x_{1}{ }^{\prime} x_{2}{ }^{\prime}$ & $x_{1}{ }^{\prime} y^{\prime}$ & $x_{2} y^{\prime}$ \\
\hline 1 & 3.12 & 79.6 & 0.9333 & 6336.16 & 0.8711 & 74.2933 & 248.352 & 2.9120 \\
\hline 2 & 2.02 & -9.4 & -3.0667 & 88.36 & 9.4044 & 28.8267 & -18.988 & -6.1947 \\
\hline 3 & -0.88 & -121.4 & -2.0667 & 14737.96 & 4.2711 & 250.8933 & 106.832 & 1.8187 \\
\hline 4 & 3.22 & 108.6 & 8.9333 & 11793.96 & 79.8044 & 970.1600 & 349.692 & 28.7653 \\
\hline 5 & 3.42 & 202.6 & 5.9333 & 41046.76 & 35.2044 & 1202.0933 & 692.892 & 20.2920 \\
\hline 6 & -3.18 & -83.4 & -1.0667 & 6955.56 & 1.1378 & 88.9600 & 265.212 & 3.3920 \\
\hline 7 & -1.28 & -93.4 & 3.9333 & 8723.56 & 15.4711 & -367.3733 & 119.552 & -5.0347 \\
\hline 8 & -2.88 & -176.4 & -4.0667 & 31116.96 & 16.5378 & 717.3600 & 508.032 & 11.7120 \\
\hline 9 & 0.22 & -91.4 & -0.0667 & 8353.96 & 0.0044 & 6.0933 & -20.108 & -0.0147 \\
\hline 10 & 1.02 & -41.4 & -1.0667 & 1713.96 & 1.1378 & 44.1600 & -42.228 & -1.0880 \\
\hline 11 & -1.18 & -56.4 & -4.0667 & 3180.96 & 16.5378 & 229.3600 & 66.552 & 4.7987 \\
\hline 12 & -2.88 & -126.4 & -3.0667 & 15976.96 & 9.4044 & 387.6267 & 364.032 & 8.8320 \\
\hline 13 & -0.98 & 326.6 & -1.0667 & 106667.56 & 1.1378 & -348.3733 & -320.068 & 1.0453 \\
\hline 14 & 0.12 & -99.4 & -7.0667 & 9880.36 & 49.9378 & 702.4267 & -11.928 & -0.8480 \\
\hline 15 & 0.12 & 181.6 & 6.9333 & 32978.56 & 48.0711 & 1259.0933 & 21.792 & 0.8320 \\
\hline
\end{tabular}

Based on the data from Table 2, the potential forecast data can easily be calculated and compared, which is helpful for quality engineers to make a rational decision.

Without doubt, the two calculations can be amalgamated as follows:

$$
\bar{x}_{1}=\sum_{i=1}^{15} x_{1(i)}=1441.4
$$




$$
\bar{x}_{2}=\sum_{i=1}^{15} x_{2(i)}=19.0667
$$

Based on Equation (6) and (7), the general coefficient of forecasting equation can be calculated in the following:

$$
\begin{gathered}
a_{1}=\frac{\sum x_{1}^{\prime} y^{\prime} \cdot \sum\left(x_{2}^{\prime}\right)^{2}-\sum x_{1}^{\prime} x_{2}^{\prime} \cdot \sum x_{2}^{\prime} y^{\prime}}{\sum\left(x_{1}^{\prime}\right)^{2} \cdot \sum\left(x_{2}^{\prime}\right)^{2}-\sum x_{1}^{\prime} x_{2}^{\prime} \cdot \sum x_{1}^{\prime} x_{2}^{\prime}}=\frac{2329.62 \times 288.9333-5245.6 \times 71.22}{299551.6 \times 288.9333-5245.6 \times 5245.6}=0.0051 \\
a_{2}=\frac{\sum x_{2}^{\prime} y^{\prime} \cdot \sum\left(x_{1}^{\prime}\right)^{2}-\sum x_{1}^{\prime} x_{2}^{\prime} \cdot \sum x_{1}^{\prime} y^{\prime}}{\sum\left(x_{1}^{\prime}\right)^{2} \cdot \sum\left(x_{2}^{\prime}\right)^{2}-\sum x_{1}^{\prime} x_{2}^{\prime} \cdot \sum x_{1}^{\prime} x_{2}^{\prime}}=\frac{71.22 \times 299551.6-5245.6 \times 2329.62}{299551.6 \times 288.9333-5245.6 \times 5245.6}=0.1544
\end{gathered}
$$

Thus, the predicted data can be given by Equation (8):

$$
a_{0}=\bar{y}-a_{1} \bar{x}_{1}+a_{2} \bar{x}_{2}=94.38-0.0051 \times 1441.4+0.1544 \times 19.0667=90.0105
$$

Then, the many Yuan linear return forecast model is defined as:

$$
y=a_{0}+a_{1} x_{1}+a_{2} x_{2}=90.0105+0.0051 x_{1}+0.1544 x_{2}
$$

With the help of the Equation (9), the multiple correlations efficient are presented in the following:

$$
R=\sqrt{1-\frac{22.8146}{68.464}}=0.8166
$$

Applying the Equation (10), we can get the value of $\mathrm{F}$ testing is given as:

$$
F=\frac{0.8166 \times 0.8166 / 2}{(1-0.8166 \times 0.8166) /(15-2-1)}=12.0053
$$

Using database partition groups, we can determine across by comparing the size of the data, which is show in the following:

$$
F=12.0053>F_{\alpha}=6.36
$$

Thus, let us suppose that the MTBF is 1270 , and product development lifecycle is 13 , we can calculated predictive values in the following equation:

$$
y=90.0105+0.0051 x_{1}+0.1544 x_{2}=90.0105+0.0051 \times 1270+0.1544 \times 13=98.5
$$

At last, we can get the predicted value for reliability of system is $98.5 \%$.

In addition to theoretically of research and summary, the product quality in CNC machining center has been kept in an excellent condition. The product quality there has attained the first-level quality and the quality of the entire machine is also higher than the national standard.

Compared with other models through the multiple linear regression analysis, the accuracy of the approved model increases significantly when the process parameters and product properties was included. The idea of the approved approach is to present a list of variables with their weights of importance in product quality prediction. 


\section{Conclusion}

The purpose of this article was to present a new personalized forecasting system for complex product quality as well as introduce empirical evidence on the importance of predictive variables of predictive control model. Predicting complex product quality is crucial to quality management, as well as to manufacturing firms seeking to mitigate potential losses. This paper has presented a personalized forecasting system for complex product quality based on predictive control model of multiple linear regression analysis. And the personalized forecasting system anticipates that past quality cost data collected from product designing and manufacturing process may be correlated in some nonlinear way and may not lend them to fit only one linear equation within a given error bound. The results of this forecasting process were a set of stepwise linear equations and their applicable boundaries.

In the next stage of this project, models will be developed to serve as practical tools for quality engineers and enterprises' decision-makers. The approved system and methods may affect the predictive accuracy of the linear equation and further study is needed to quantify the effect. Future research will also investigate the use of other control models and other personalized forecasting system to improve forecasts further.

\section{Acknowledgments}

This work was supported by the National Natural Science Foundation, China (No.71301120), the Provincial Natural Science Foundation, Zhejiang, China (No.LY13G010002).

\section{References}

[1] S. Tyagi, A. Choudhary, X. Cai and K. Yang, "Value stream mapping to reduce the lead-time of a product development process", International Journal of Production Economics, vol. 160, (2015), pp. 202-212.

[2] G. J. Besseris, "Improving quality cost performance with qualimetrical methods: A case from a highspeed packaging process", International Journal of Advanced Manufacturing Technology, vol. 69, (2013), pp. 1775-1789.

[3] S. S. Durmusoglu, "Merits of task advice during new product development: Network centrality antecedents and new product outcomes of knowledge richness and knowledge quality", Journal of Product Innovation Management, vol. 30, (2013), pp. 487-499.

[4] A. Garstenauer, T. Blackburn and B. Olson, "A knowledge management based approach to quality management for large manufacturing organizations", EMJ - Engineering Management Journal, vol. 26, (2014), pp. 47-58.

[5] Q. Le, Z. Sha, and J. H. Panchal, "A generative network model for product evolution", Journal of Computing and Information Science in Engineering, vol. 14, (2014).

[6] J. Lee, K. C. Kang, P. Sawyer and H. Lee, "A holistic approach to feature modeling for product line requirements engineering", Requirements Engineering, vol. 19, (2014), pp. 377-395.

[7] A. P. Longstaff, S. Fletcher, S. Parkinson and A. Myers, "The role of measurement and modelling of machine tools in improving product quality", International Journal of Metrology and Quality Engineering, vol. 4, (2013), pp. 177-184.

[8] M. J. North and C. M. MacAl, "Product and process patterns for agent-based modelling and simulation", Journal of Simulation, vol. 8, (2014), pp. 25-36.

[9] A. Musuku, A. Tan, K. Awaiye and F. Trabelsi, "Comparison of two-concentration with multiconcentration linear regressions: Retrospective data analysis of multiple regulated LC-MS bioanalytical projects", Journal of Chromatography B: Analytical Technologies in the Biomedical and Life Sciences, vol. 934, (2013), pp. 117-123.

[10] B. Corbett, B. MacDonald and P. Mhaskar, "Model predictive quality control of polymethyl methacrylate", IEEE Transactions on Control Systems Technology, vol. 23, (2015), pp. 687-692.

[11] K. R. Reddi and Y. B. Moon, "Modelling engineering change management in a new product development supply chain", International Journal of Production Research, vol. 51, (2013), pp. 52715291

[12] M. E. Sosa, J. Mihm and T. R. Browning, "Linking cyclicality and product quality, Manufacturing and Service Operations Management", vol. 15, (2013), pp. 473-491.

[13] A. Dhurandhar and M. Petrik, "Efficient and accurate methods for updating generalized linear models with multiple feature additions", Journal of Machine Learning Research, vol. 15, (2014), pp. 2607-2627. 
[14] G. Pietila and T. C. Lim, "Sound quality preference modeling using a nested artificial neural network architecture", Noise Control Engineering Journal, vol. 63, (2015), pp. 138-151.

[15] S. Aumi, B. Corbett, T. Clarke-Pringle and P. Mhaskar, "Data-driven model predictive quality control of batch processes", AIChE Journal, vol. 59, (2013), pp. 2852-2861.

[16] R. Dubey and A. Gunasekaran, "Exploring soft TQM dimensions and their impact on firm performance: Some exploratory empirical results", International Journal of Production Research, vol. 53, (2015), pp. 371-382.

[17] S. Hoque, B. Farouk and C. N. Haas, "Multiple linear regression model approach for aerosol dispersion in ventilated spaces using computational fluid dynamics and dimensional analysis", Journal of Environmental Engineering, vol. 136, (2010), pp. 638-649.

[18] U. Sofowote, Y. Su, M. M. Bitzos and A. Munoz, "Improving the correlations of ambient tapered element oscillating microbalance PM2.5 data and SHARP 5030 Federal Equivalent Method in Ontario: A multiple linear regression analysis", Journal of the Air and Waste Management Association, vol. 64, (2014), pp. 104-114.

[19] L. M. L. Laurens and E. J. Wolfrum, "High-throughput quantitative biochemical characterization of algal biomass by NIR spectroscopy; Multiple linear regression and multivariate linear regression analysis", Journal of Agricultural and Food Chemistry, vol. 61, (2013), pp. 12307-12314.

[20] Z. B. Wu, "Empirical analysis for personal insurance market demand in Heilongjiang province", Journal of Harbin University of Science and Technology, vol. 16, (2011), pp. 124-128. 